\title{
Síndrome de trisomía 18: informe de un caso clínico
}

\author{
Trisomy 18 syndrome: a case report
}

\section{David Alexander Montoya Reales, ${ }^{1}$ Carol Gabriela Lanza, ${ }^{1}$ Javier David Almendares. ${ }^{2}$}

${ }^{1}$ Médico General, egresado de la Facultad de Ciencias Médicas, Universidad Nacional Autónoma de Honduras.

${ }^{2}$ Pediatra, Centro de Salud Villadela, Comayagüela.

RESUMEN. Antecedentes: El Síndrome de Edwards o trisomía 18, es una anomalía cromosómica o aneuploidía caracterizada por la presencia de una copia adicional del cromosoma 18. Se asocia con una alta tasa de mortalidad, siendo muy escasas las probabilidades de supervivencia más allá de las semanas o del año de edad. Sin embargo, existen casos que llegan a sobrevivir más tiempo, pero con retraso mental severo, problemas de salud y de desarrollo, y por ende una calidad de vida muy baja. En este artículo se informa un caso de trisomía 18 de larga sobrevida con el propósito de aportar información sobre la etiología, el fenotipo, la sobrevida y el consejo genético. Descripción de Caso: Paciente de sexo femenino nacida en 2010 con un cariotipo realizado en 20 células que mostraron $47, X X+18$ en todas las metafases. Con talla y peso bajos para su edad, facies dismórficas, retardo severo del desarrollo psicomotor y cognitivo, imposibilidad para alimentarse, ausencia de lenguaje verbal y sordera neurosensorial. Conclusión: El conocimiento de las manifestaciones clínicas y el pronóstico del paciente con trisomía 18 es de gran importancia para el cuidado neonatal y la toma de decisiones para realizar procedimientos invasivos, como cirugía de corazón o resucitación cardiopulmonar. El diagnóstico temprano es importante para la toma de decisiones médicas.

Palabras clave: Aberraciones cromosómicas, Síndrome de Edwards, Síndrome de la Trisomía 18, Trisomía.

\section{INTRODUCCIÓN}

El síndrome de trisomía 18 (TS18) o síndrome de Edwards (SE) es un trastorno cromosómico autosómico que se caracteriza por la presencia de un cromosoma 18 extra completo. Además, se han reportado en la literatura casos de T18 en mosaico y de trisomía parcial. ${ }^{1}$ Es la segunda trisomía autosómica más frecuente en recién nacidos. ${ }^{2}$ Su incidencia es de 1/3000$1 / 8000$ nacidos vivos, pero su incidencia depende del diagnóstico prenatal. ${ }^{3}$ El $80 \%$ de los casos son niñas. Cualquier mujer presenta el riesgo de tener un niño con esta anormalidad. La edad materna avanzada está relacionada con frecuencia con la incidencia de esta condición. ${ }^{4}$

El patrón del síndrome comprende varios patrones reconocibles de anomalías mayores y menores, discapacidad psicomotora y cognitiva significativa. ${ }^{5}$ TS18 se caracteriza clínicamente por un amplio espectro de manifestaciones, con más de 130 hallazgos diferentes descritos. El pronóstico es considerado pobre. ${ }^{6}$ TS18 no está asociado con una supervivencia a largo termino, $5-10 \%$ de los niños nacidos vivos sobreviven más de un año. ${ }^{7}$ En este artículo informamos un caso de TS18 de larga sobrevida con el propósito de aportar información sobre la etiología, el fenotipo, la sobrevida y el consejo genético. El caso fue evaluado y documentado en el año 2016 y por su importancia y lecciones aprendidas, se decide publicar.

\section{DESCRIPCIÓN DEL CASO}

Paciente de sexo femenino, nacida en año 2010 y evaluada a los 5 años de edad. Al ser evaluada se documentó talla de $101.5 \mathrm{~cm}$, peso de $9.5 \mathrm{~kg}$, perímetro cefálico de $43 \mathrm{~cm}$ y perímetro torácico de $49 \mathrm{~cm}$. Los hallazgos dismórficos presentes al examen físico incluyeron los descritos a continuación. Craneofaciales: Microcefalia, occipucio prominente, crestas supraorbitarias hipoplásicas, fisuras palpebrales pequeñas, inclinación mongoloide, epicanto, pabellones auriculares malformados y con implantación baja, atresia de coanas, limitación de la apertura bucal, micrognatia. Piel: Hirsutismo en espalda y frente. Tórax: Esternón corto, pectumexcavatum. Manos y pies: Manos trisómicas, ausencia de pliegue falángico distal. Genitourinario: Hipoplasia de labios mayores. Además, se identificó hipoplasia 


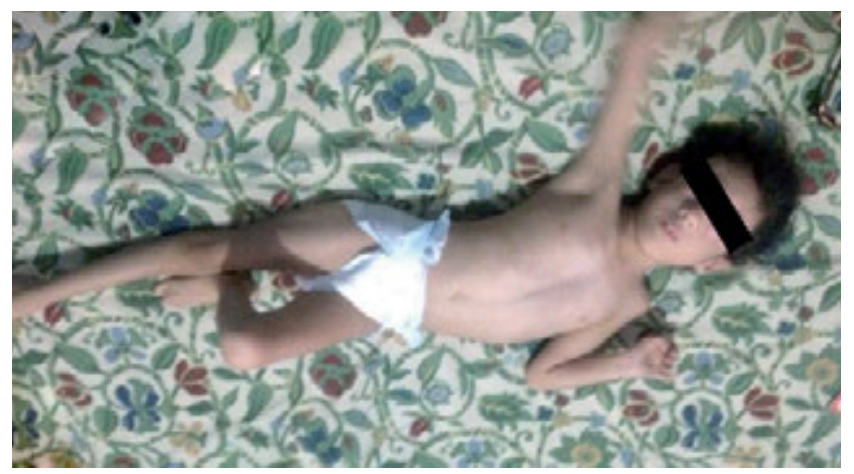

Figura 1. Paciente de 5 años de edad con Síndrome de Trisomía 18. Se puede observar hipoplasia del tejido muscular esquelético, tejido subcutáneo y panículo adiposo e hipertonía.

del tejido muscular esquelético, tejido subcutáneo y panículo adiposo, hipertonía, marcada deficiencia mental y respuesta disminuida al sonido (Figura 1).

La paciente es hija de padres no consanguíneos, sin antecedentes familiares de enfermedades agudas, crónicas, infecciosas o genéticas de importancia. Madre al momento del embarazo con 28 años de edad, primigesta, con control prenatal sin observar en ultrasonidos alteraciones, no exposición a teratógenos. Producto de parto vaginal inducido por ruptura prematura de membranas. Al momento del nacimiento APGAR normal, peso: $1800 \mathrm{gr}$, perímetro cefálico: $41 \mathrm{~cm}$, con varios hallazgos dismórficos, hospitalizada durante 18 días en incubadora por bajo peso al nacer. Al mes de edad se realizó cariotipo con bandas $\mathrm{G}$, que mostró $47, X X+18$. Se analizaron 20 células en metafase y en todas se encontró un cromosoma 18 adicional.

En el transcurso de su vida ha presentado retraso en su crecimiento y desarrollo físico y cognitivo. A los cinco años de edad se informó de varios aspectos importantes tales como no controlar esfínteres, balbucear, no sostener la cabeza, no poder alimentarse sola. Presentó convulsiones a los tres meses de edad, fue manejada con ácido valproico y se informó que la paciente no ha presentado convulsiones en los últimos tres años. Por episodios de neumonía a repetición se sospechó reflujo gastroesofágico por lo que fue manejada con lanzoprazol. Posterior a este manejo, no presentó nuevos episodios de neumonía. Se realizó electroencefalograma que demostró actividad convulsiva generalizada y moderada. La TAC cerebral al año de edad reportó atrofia cerebral. TAC de tórax mostró cardiomegalia y fibrosis pulmonar congénita. La radiografía de columna reportó rotoescoliosis. El ecocardiograma demostró Enfermedad de Ebstein, foramen ovale permeable, que es manejada con digoxina. Se obtuvo consentimiento informado de su madre para publicar el caso clínico.

\section{DISCUSIÓN}

La TS18 fue descrita por primera vez en 1960 por Edwards y colaboradores quienes reportaron un recién nacido con múltiple malformaciones y deficiencia cognitiva. Los autores reportaron un síndrome de trisomía nuevo, el cual fue nombrado como trisomía 17-18. Después de la descripción del síndrome, en
1960, Smith y colaboradores demostraron que el cromosoma adicional era el cromosoma 18. La TS18 es una de las trisomías autosomal más frecuente observada al nacimiento. ${ }^{8}$

Es bien reconocido que los embarazos con productos con T18 tienen un alto riesgo de pérdida fetal y parto con un feto muerto; además, actualmente la mayoría de los diagnósticos son realizados en el periodo prenatal basado en la edad materna o marcadores en el suero materno y amniocentesis, seguido por la interrupción del embarazo en un porcentaje importante de casos. La prevalencia al nacimiento es mayor en sexo femenino comparado con sexo masculino, un predominio de 3 a 1 a favor de las mujeres, pero esta discordancia no está presente si la razón del sexo es calculada entre los embarazos electivamente terminados. ${ }^{9,10}$ Además, la frecuencia de pérdida fetal es mayor en fetos del sexo masculino comparado con sexo femenino. También, las mujeres nacidas vivas muestran una mejor sobrevida comparada con los hombres. ${ }^{9}$

Según estudios la TS18 se presenta de tres maneras: Trisomía 18 total, en un $95 \%$ de los casos, es decir que se presentan tres copias exactas y completas del cromosoma 18 en cada célula del organismo; Trisomía 18 parcial en un $2 \%$ de los casos, en los cuales existirán dos cromosomas 18 más una parte extra de otro cromosoma 18; Trisomía 18 con mosaicismo, en un $3 \%$ de los casos, lo cual quiere decir que la trisomía puede no presentarse en todas las células el organismo. El mecanismo involucrado en la mayoría de los casos de trisomía completa del cromosoma 18 es una no disyunción que se da en la gametogénesis, llevando al defecto de la no segregación o separación normal de los cromosomas. De manera general esa no disyunción sucede en la ovogénesis, en un $95 \%$ de los casos aproximadamente, siendo más rara su aparición en la espermatogénesis. ${ }^{11}$

La presencia de doble aneuploidía (dos anormalidades cromosómicas) en una misma persona, es un fenómeno raro en recién nacidos vivos, pero frecuentemente observado en abortos espontáneos. Los casos reportados de doble aneuploidía involucra autosomas y cromosomas sexuales. Las asociaciones de síndromes por aneuploidía que se han descrito incluyen los Síndromes Edwards-Down, Down-Klinefelter, Down-Turner mosaico, Down-XYY, Patau-Klinefelter, Edwards-Turner mosaico, Edwards-XXX, Edwards-Klinefelter y Edwards-XYY.12

In útero el síndrome de TS18 se sospecha en función de la identificación de hallazgos ecográficos. El aumento en la translucencia nucal y la ausencia de hueso nasal (también usados en el síndrome de Down y el síndrome de Patau) se observan en el $66 \%$ de los fetos con TS18. Otros marcadores ecográficos, como flujo reverso del ductus venoso y regurgitación en la válvula tricuspídea, aumentan la detección hasta un $83.3 \%$. Algunos protocolos integran marcadores ecográficos y bioquímicos en el primer trimestre y pruebas bioquímicas en el segundo trimestre, alcanzando tasas de detección hasta del $78 \%$; sin embargo, esta prueba de tamizaje integrada está en desuso. ${ }^{1}$

Los fetos con TS18 pueden presentar anomalías anatómicas únicas o múltiples. Algunas se pueden detectar en la ecografía del primer trimestre, pero con mayor probabilidad de observarse en el segundo o tercer trimestre del embarazo; estas son: onfalocele, postura anormal de las manos (desviación radial 
o ulnar), mega vejiga, anormalidades cardiacas (usualmente las detectables en la vista de 4 cámaras), megacisterna magna, retardo en el crecimiento intrauterino (siendo frecuente su inicio en el segundo trimestre del embarazo), polihidramnios, cráneo en forma de fresa, quiste de plexo coroideo, dedos superpuestos (conocido como mano trisómica, desviación de los dedos hacia la línea media), arteria umbilical única. ${ }^{1} \mathrm{~A}$ la paciente cuyo caso se está informando, no se le encontraron anomalías en la ecografía.

El reciente desarrollo de pruebas prenatales no invasivas de secuencia paralela masiva de ADN fetal de células libres (cffDNA por sus siglas en inglés), circulando en sangre periférica materna, representa una nueva era de tamizaje prenatal. Estos fragmentos de ADN son derivados de células de citotrofoblasto apoptótico. Varios estudios muestran que el análisis de cffDNA puede detectar $99 \%$ de los casos de trisomía 21 , $97 \%$ de trisomía 18 y $92 \%$ de trisomía 13 , con falsos positivos de aproximadamente $0.1 \%, 0.2 \%$ y $0.2 \%$ respectivamente. Sin embargo, estos estudios fueron retrospectivos y realizados en población con riesgo alto y edad materna avanzada ( $\geq 35$ años de edad). ${ }^{13-15}$ Estos estudios complementarios no se le realizaron a la paciente cuyo caso se está informando, dado que no se dispone de ellos en la institución.

Las manifestaciones clínicas más frecuentes de TS18 se describen en el Cuadro 1, aunque ninguna de ellas es patogno- mónica para la enfermedad. ${ }^{10,16}$ El diagnóstico se confirma con la realización de cariotipo. El más utilizado es el preparado con la tinción de Giemsa, aunque también se puede realizar FISH o hibridación genómica comparativa por microarreglos, todos los cuales se pueden realizar en diagnóstico prenatal o posnatal. ${ }^{1}$ Los hallazgos fenotípicos son variables. No obstante, no se evidencia una correlación entre el porcentaje de células trisómicas en leucocitos o fibroblastos y los hallazgos clínicos encontrados. ${ }^{17} \mathrm{~A}$ la paciente cuyo caso se está informando, el diagnóstico fue realizado con cariotipo con bandas $\mathrm{G}$, que mostro $47, \mathrm{XX}$ + 18. La TS18 es una condición a menudo denominada "incompatible con la vida" o "anomalía letal". Los futuros padres y los padres con recién nacidos con un diagnóstico posterior al parto deben proporcionar intervenciones médicas mínimas y, si el recién nacido sobrevive, esperar una calidad de vida limitada. ${ }^{18}$

La mayoría de los fetos con T18 no sobreviven hasta el final del periodo gestacional. Los que nacen vivos tienen un pobre pronóstico. La supervivencia media reportada en la literatura es de 2.5 a 14.5 días. En general, $55-65 \%$ de los recién nacidos afectados mueren durante la primera semana, $90 \%$ en seis meses, y solo $5-10 \%$ alcanza el año de vida. ${ }^{8}$ Los pacientes con supervivencia a largo plazo de la trisomía 18 exhiben retraso grave del desarrollo psicomotor. ${ }^{19}$ Se está prestando cada vez más atención a los aspectos éticos de la trisomía 18, que implica

Cuadro 1. Manifestaciones clínicas frecuentes del Síndrome de Trisomía 18 (Adaptado de referencia 10).

\begin{tabular}{|c|c|c|}
\hline \multirow{2}{*}{$\begin{array}{l}\text { Sistema } \\
\text { Craneofacial }\end{array}$} & \multicolumn{2}{|c|}{ Manifestaciones Clínicas } \\
\hline & $\begin{array}{l}\text { - Microcefalia } \\
\text { - Occipucio prominente } \\
\text { - Cráneo dolicocéfalo } \\
\text { - Crestas supraorbitarias hipoplásicas } \\
\text { - Fisuras palpebrales pequeñas } \\
\text { - Inclinación mongoloide } \\
\text { - Epicanto } \\
\text { - Coloboma, hendidura en el iris }\end{array}$ & $\begin{array}{ll}\text { - } & \text { Pabellones craneales malformados y con implantación baja } \\
\text { - } & \text { Atresia de coanas } \\
\text { - } & \text { Limitación de la apertura bucal } \\
\text { - } & \text { Micrognatia } \\
\text { - } & \text { Paladar ojival } \\
\text { - } & \text { Macrostomía } \\
\text { - } & \text { Labio leporino o fisura palatina }\end{array}$ \\
\hline Cardiovascular & - Cardiopatías congénitas, v.g. Comunicación interauricular & $\begin{array}{ll}\text { - } & \text { Conducto arterial persistente } \\
\text { - } & \text { Comunicación interventricular }\end{array}$ \\
\hline Tórax & $\begin{array}{l}\text { - Esternón corto } \\
\text { - Tórax en quilla }\end{array}$ & - Pectum excavatum \\
\hline Abdomen & $\begin{array}{l}\text { - Hernia inguinal, hernia umbilical } \\
\text { - Diástasis de los músculos rectos }\end{array}$ & $\begin{array}{ll}\text { - } & \text { Onfalocele } \\
\text { - } & \text { Hernia diafragmática }\end{array}$ \\
\hline Genitourinario & $\begin{array}{l}\text { - Hipoplasia de labios mayores } \\
\text { - Criptorquidia }\end{array}$ & $\begin{array}{ll}\text { - } & \text { Riñón poliquístico } \\
\text { - } & \text { Riñón ectópico } \\
\text { - } & \text { Hidronefrosis }\end{array}$ \\
\hline Pelvis y cadera & - Pelvis pequeña, limitación de la abducción de la cadera & - Luxación de cadera \\
\hline Manos y pies & $\begin{array}{l}\text { - Manos trisómicas: es decir manos cerradas en puño con sobre- } \\
\text { posición de dedos } \\
\text { - Ausencia de pliegue falángico distal } \\
\text { - Hipoplasia ungueal }\end{array}$ & $\begin{array}{ll}\text { - } & \text { Pulgar hipoplásico o ausente } \\
\text { - } & \text { Aplasia radial } \\
\text { - } & \text { Sindactilia } \\
\text { - } & \text { Dermatoglifos muestran figuras de arcos en todos los dedos }\end{array}$ \\
\hline Piel & - Cutis marmorata & - Hirsutismo en espalda y frente \\
\hline Sistema nervioso & $\begin{array}{l}\text { - Anomalía vertebral } \\
\text { - Escoliosis } \\
\text { - Parálisis facial } \\
\text { - Mielinización escasa }\end{array}$ & $\begin{array}{ll}\text { - } & \text { Defectos de cuerpo calloso } \\
\text { - } & \text { Hidrocefalia } \\
\text { - } & \text { Mielomeningocele } \\
\text { - } & \text { Megacisterna magna }\end{array}$ \\
\hline Otros & $\begin{array}{l}\text { - Marcada deficiencia mental después del período prenatal } \\
\text { - Respuesta disminuida al sonido } \\
\text { - Hipotonía inicial que evoluciona a hipertonía }\end{array}$ & $\begin{array}{ll}\text { - } & \text { Hipoplasia tímica } \\
\text { - } & \text { Atresia esofágica } \\
\text { - } & \text { Fístula traqueoesofágica } \\
\text { - } & \text { Trombocitopenia }\end{array}$ \\
\hline
\end{tabular}


la cuestión de si resucitar o no a los bebés afectados. En particular, la insuficiencia cardíaca, la apnea y / o la insuficiencia respiratoria son las principales causas de muerte en los lactantes con trisomía 18. Sin embargo, se ha demostrado que la aplicación de intervenciones médicas y quirúrgicas para los lactantes con trisomía 18 ayuda a su supervivencia a largo plazo. Por lo tanto, hay un debate en curso sobre el alcance de las intervenciones apropiadas para los recién nacidos con trisomía $18 .^{20}$

En nuestro caso, a pesar de las enfermedades concomitantes que presentó la paciente ha logrado sobrepasar las expectativas de vida. Su familia, en especial su madre dedica esmero a su cuidado, acudiendo a sus citas médicas y de fisioterapia regularmente. Adicionalmente, es muy importante proporcionar consejo genético, en el que se explique que para una pareja con un niño con T18 libre y completa la probabilidad de repetición en el siguiente embarazo es del $1 \%{ }^{1}$ La última fecha de contacto con la familia de la paciente fue en el año 2017.
En conclusión, son muy pocos los casos reportados que sobrepasan los 5 años de edad. En los casos de TS18 la mortalidad in útero y neonatal es alta. No obstante, la sobrevida mejora cuando las anomalías congénitas son menores, se da soporte cuando se requiere en la unidad de cuidados intensivos y se realizan cirugías paliativas o correctivas. Las características clínicas in útero y en el recién nacido han sido bien descritas. El conocimiento de las manifestaciones clínicas y el pronóstico del paciente con T18 son de gran importancia para el cuidado neonatal y la toma de decisiones para realizar procedimientos invasivos, como cirugía de corazón o resucitación cardiopulmonar. El diagnóstico temprano es importante para la toma de decisiones médicas.

\section{CONTRIBUCION DE LOS AUTORES}

Todos los autores contribuyeron en igual medida a la realización del artículo, así como en la revisión y aprobación de la versión final.

\section{REFERENCIAS}

1. Saldarriaga W, Rengifo-Miranda H, Ramírez-Cheyne J. Síndrome de trisomía 18. Reporte de un caso clínico. Rev chil Pediatr. 2016;87(2):129-136.

2. Imai K, Uchiyama A, Okamura T, Ago M, Suenaga $\mathrm{H}$, Sugita E, et al. Differences in mortality and morbidity according to gestational ages and birth weights in infants with trisomy 18. Am J Med Genet. 2015; 167(11): 2610-2617.

3. Bustillos-Villalta K, Quiñones Campos M. Síndrome de Edwards de larga supervivencia: efecto del tratamiento rehabilitador integral. Rev Med Hered. 2014:25(2):89-92.

4. Tarcă E, Plămădeală $P$, Savu B. Plurimalformative syndrome associating trisomy 18 and omphalocele. Case report and review of the literature. Rom J Morphol Embryol. 2014; 55(1):209-213.

5. Mudaliyar US, Mudaliyar SU. Strawberry skull in Edwards syndrome. BJR Case Rep. 2017;3(4):20170045.

6. Denardin D, Savaris FE, da Cunha AC, Betat Rda S, Telles JA, Targa LV et al. Retrospective cohort of trisomy 18 (Edwards syndrome) in southern Brazil. Sao Paulo Med J. 2015; 133(4):320-325.

7. Palomaki GE, Deciu C, Kloza EM, Lambert Messerlian GM, Haddow JE, Neveux LM, et al. DNA sequencing of maternal plasma reliably identifies trisomy 18 and trisomy 13 as well as Down syndrome: an international collaborative study. Genet Med. 2012;14(3):296-305.

8. Rosa RF, Rosa RC, Zen PR, Graziadio C, Paskulin GA. Trisomy 18: review of the clinical, etiologic, prognostic, and ethical aspects. Rev Paul Pediatr. 2013; 31(1):111-120.

9. Cereda A, Carey JC. The trisomy 18 syndrome. Orphanet J Rare Dis. 2012; 7:81.

10. Pachajoa $H$. Doble aneuploidía (trisomía $X$, trisomía 18 ) en una recién nacida con fenotipo de trisomía 18. Arch Argent Pediatr. 2013;111(4):e101e104.

11. Villalba Herrera EW, Roca Cruz CA. Sindrome de Edwards. Rev Act Clin Med. 2014;45:2384-2388

12. Gómez Valencia L, Briceño-González M, Morales Hernández A, RiveraAngles MM, Cornelio-García RM, Toledo Ocampo E. Síndrome de Edwards mosaico con doble aneuploidia: $46, X X / 47, X X X / 47, X X,+18 / 48$, XXX, +18. Salud en Tabasco 2011; 17(1-2):41-45.

13. Hochstenbach R, Page Christiaens G, van Oppen A, Lichtenbelt K, van Harssel J, Brouwer T, et al. Unexplained false negative results in noninvasive prenatal testing: two cases involving trisomies 13 and 18. Case Rep Genet. 2015;2015 (926545).

14. Ke WL, Zhao WH, Wang XY. Detection of fetal cell-free DNA in maternal plasma for Down syndrome, Edward syndrome and Patau syndrome of high-risk fetus. Int J Clin Exp Med 2015;8:9525-9530.

15. Quezada MS, Gil MM, Francisco C, Oròsz G, Nicolaides KH. Screening for trisomies 21,18 and 13 by cell-free DNA analysis of maternal blood at
10-11 weeks' gestation and the combined test at 11-13 weeks. Ultrasound Obstet Gynecol. 2015;45(1):36-41.

16. Ferreira de Souza LM, Galvão E Brito Medeiros A, Júnior JPR, de Melo AN, Dias SAMM. Long Survival of a Patient with Trisomy 18 and Dandy-Walker Syndrome. Medicina (Kaunas) 2019;55(7):352.

17. Cammarata-Scalisi F, Lacruz-Rengel MA, Araque D, Da Silva G, Avendaño A, Callea M, et al. Trisomía 18 en mosaico. Serie de casos. Arch Argent Pediatr. 2017;115(3):e183-e186.

18. Bruns DA. Developmental status of 22 children with trisomy 18 and eight children with trisomy 13: Implications and recommendations. Am J Med Genet A. 2015;67A(8):1807-1815.

19. Imataka $G$, Suzumura $H$, Arisaka $O$. Clinical features and survival in individuals with trisomy 18: A retrospective one-center study of 44 patients who received intensive care treatments. Mol Med Rep. 2016;13: 2457-2466.

20. Taira R, Inoue H, Sawano T, Fujiyoshi J, Ichimiya Y, Torio M, et al. Management of apnea in infants with trisomy 18. Dev Med Child Neurol. 2020;62(7):874-878. Epub 2019 Nov 25.

ABSTRACT. Background: Edwards Syndrome or trisomy 18, is a chromosomal aneuploidy characterized by the presence of an extra copy of chromosome 18. This syndrome is associated with a high mortality rate, with very few chances of survival beyond the week or year of age. However, there are cases that survive longer but with severe mental retardation, health and development and therefore very low quality life. In this paper we report a case of trisomy 18 of long survival with the purpose to provide information about aetiology, phenotype, survival and genetic counselling. Case Description: A 5 -year-old female patient with one karyotype performed on 20 cells showing $47 X X+18$ in all metaphases. She presented with weight and height low for age, dysmorphic facies, severe psychomotor retardation and cognitive disability, inability to feed, lack of verbal language and sensorineural hearing loss. Conclusion: The knowledge of the clinical features and prognosis of patients with trisomy 18 is of great importance regarding the neonatal care and the decision of performing invasive procedures, such as heart surgery or cardiopulmonary resuscitation. The early diagnostic confirmation is important for making medical decisions.

Keywords: Chromosome aberrations, Edwards syndrome, Trisomy 18 Syndrome, Trisomy. 